\title{
Multi-faceted approaches toward unravelling complex ecological networks
}

\author{
Toshiyuki Namba ${ }^{1}$
}

Received: 16 February 2015/Accepted: 3 March 2015/Published online: 17 March 2015

(C) The Author(s) 2015. This article is published with open access at Springerlink.com

\begin{abstract}
The main theme of this Special Feature is the complexity-stability relationship and diversity of interaction types. Five articles by leading authors are submitted. Studies on the relationship between complexity and stability have a long history of 40 years. Effect of multiple interaction types on structure and dynamics of an ecological network is a recent important subject to be resolved. In this preface, I briefly review the history of research on complexity-stability relationship till the idea of diversity of interaction types appears.
\end{abstract}

Keywords Complexity - Diversity - Ecological network · Interaction strength $\cdot$ Interaction type $\cdot$ Stability

\section{Introduction}

This review was written as a preface for the Special Feature "Unravelling ecological networks: complexitystability relations and diversity of interaction types". The main theme of the Special Feature is the complexity-stability relationship and diversity of interaction types. Five articles by leading authors are submitted; "Comparing the conservatism of ecological interactions in plant-pollinator and plant-herbivore networks" by Colin Fontaine and

\footnotetext{
Toshiyuki Namba

tnamba@b.s.osakafu-u.ac.jp

1 Osaka Prefecture University, Sakai, Osaka, Japan
}

Elisa Thébault; "Interaction-type diversity hypothesis and interaction strength: the condition for the positive complexity-stability effect to arise" by Michio Kondoh and Akihiko Mougi; "High-throughput DNA barcoding for ecological network studies" by Hirokazu Toju; "Analytical theory of species abundance distributions of a random community model" by Kei Tokita; "The stabilitycomplexity relationship at age 40: a random matrix perspective" by Stefano Allesina and Si Tang. To help the readers of this Special Feature to understand the contemporary significance of these works, I tried to write a brief review of the history of research on complexity-stability relationship till the idea of diversity of interaction types appears.

In the following section, "A brief history of studies on complexity-stability relationships", I will introduce a history of studies on complexity-stability relationships till ca. 2010 before the hypothesis of interaction-type diversity appears. The section is composed of 19 subsections and I tried to explain important ideas, such as indirect interactions and nonlinear dynamics, feasibility, community assemblage, food web statistics, models of food web structure, interaction strength, ecosystem functioning, metabolic theory, complex networks, secondary extinctions, and mutualistic and parasitic networks.

In the next section "Frontiers in the 2010s", I will introduce recent development in research on complexitystability relationship, focusing on diversity of interaction types and theoretical and empirical techniques. This section is composed of six subsections on communities with multiple trophic levels and multiple interaction types, random matrix theory, and sampling and processing of high throughput data. In this section, achievements by the authors in this Special Feature will be mainly introduced.

In the final section, "Coda", I will conclude this review. 


\section{A brief history of studies on complexity-stability relationships}

\section{Prologue}

The "complexity-stability" or "diversity-stability" relationship has undoubtedly been the central problem in ecology since Robert May's seminal paper in 1972 (see Goodman 1975 for a historical background of the problem). May $(1972,1973)$ extended the work by Gardner and Ashby (1970) and showed that an ecological network resting at an equilibrium point would inevitably be unstable when it became complex enough. Since this theoretical conclusion was contrary to the traditional empirical view that the more complex ecological community should be the more stable (MacArthur 1955; Elton 1958), the challenge caused the 40 years debate on the relationship between complexity and stability (Pimm et al. 1991; McCann 2000; Dunne et al. 2005; Allesina and Tang 2015). A number of theoretical works have focused on features of natural systems which were neglected in May's simple assumptions and investigated how the incorporation of biological realities would modify May's results.

\section{May's idea}

May's work was basically a linear theory assuming the existence of a feasible equilibrium (in which all populations have positive densities) in an underlying nonlinear model of community dynamics. An ecological community composed of $S$ populations is usually modelled with a continuous-time dynamical system composed of a set of $S$ autonomous (not explicitly dependent on time) ordinary differential equations. Then, stability analysis of a feasible equilibrium if it exists is done by examining eigenvalues of the community matrix, or the Jacobian matrix evaluated at the equilibrium after linearization of the nonlinear system (see Allesina and Tang 2015, for details). May's great idea was to skip the exploration of a feasible equilibrium and calculation of the Jacobian matrix, and directly proceed to construct a community matrix by randomly choosing its elements. May set all the diagonal elements to -1 and the off-diagonal elements to 0 with probability $1-C$, and with probability $C$ (called connectance, the proportion of actual links to the maximum possible links among species), he randomly chose them independently from a distribution with mean 0 and variance $\sigma^{2}$. Then, he found that the equilibrium suddenly becomes unstable with high probability when $\sigma^{2} S C$ exceeds 1 , applying the so-called Wigner's circular law (see Allesina and Tang 2015).

There were three key assumptions in May's arguments; (1) Connections (interactions) between species and their strengths are randomly chosen; (2) Existence of a feasible equilibrium in an underlying nonlinear model is implicitly assumed; (3) Stability of already assembled communities is considered instead of investigating a process of community assembly through speciation, invasion and extinction. Consequently, his criterion for stability of a feasible equilibrium was described as the product of the number of species $S$, the connectance $C$, and interaction strength $\sigma^{2}$ (variance of non-zero off-diagonal elements).

\section{Early challenges to May's conclusion}

In view of May's conclusion that a randomly constructed complex community is less likely to be stable, special features of or biologically reasonable constraints on natural ecological communities were considered to make them stable (McMurtrie 1975; Lawlor 1978; Pimm 1982). Long cycle length and hierarchical structures (McMurtrie 1975), bias toward donor dependence (De Angelis 1975), limited number of trophic levels (Pimm and Lawton 1977), low frequency and specific patterns of omnivory (Pimm and Lawton 1978; Pimm 1979a), absence of loops such that species A feeds on $\mathrm{B}$, which feeds on $\mathrm{C}$, which feeds on $\mathrm{A}$ (Gallopin 1972; Pimm 1982), high degree of compartmentalization (Pimm 1979a) and so on were examined as candidates to stabillize complex ecological communities. Yodzis (1981a) constructed community matrices from 40 real food webs and found that food webs are much more likely to be stable if the interaction strengths are chosen in accord with the patterns in natural food webs than when they are chosen randomly.

\section{Indirect interactions and nonlinear dynamics}

Those days were also a period when equilibrium theory of competition (MacArthur 1972; Cody and Diamond 1975) reached a turning point and a new era of community ecology emphasizing the importance of comparing patterns with null models (Connor and Simberloff 1979; Strong et al. 1984) and considering non-equilibrium dynamics (Wiens 1977) was in the early development stage. Understandings of indirect interactions, including keystone species (Paine 1966, 1969), trophic cascade (Paine 1980), apparent competition (Holt 1977), and exploitative competition or resource competition (Stewart and Levin 1973; León and Tumpson 1975; Hsu et al. 1978; Tilman 1980) advanced substantially and findings of complex nonlinear community dynamics, including limit cycles (May 1973), chaos (Vance 1978; Gilpin 1979), and heteroclinic cycles (May and Leonard 1975), influenced further development of community ecology and provided powerful equipment to studies on complexity-stability relationship. 


\section{Feasibility and deletion stability}

Roberts (1974) first notified that in an ecosystem interacting variables are species populations and that the equilibrium population values must be positive. He analyzed random samples from a generalized Lotka-Volterra model of up to 16 species and found that almost all feasible equilibria are stable although they are rare (see also Gilpin 1975). Then, Tregonning and Roberts (1979) considered similar systems without assuming the existence of an attainable equilibrium. If an equilibrium was unfeasible the species with the most negative equilibrium population was removed, and this process was repeated until a stable feasible equilibrium was found. Roberts and Tregonning (1980) removed subsets of any number of species from a feasible equilibrium and examined percentages of feasible equilibria in the resulting subsets. In this way, they reached a stable feasible equilibrium with considerably high diversity and found the feasibility structure of a system or robustness to large-scale disturbances (Tokita and Yasutomi 2003). Pimm (1979b, 1980) calculated species deletion stability, the probability that the resulting community remains to have a stable positive equilibrium when a species is removed. Pimm (1980) found that deletion stability decreases as the connectance or the number of species increases. After removal of a predator, further species loss was more common when the herbivores preyed upon by the predator fed on more plant species.

\section{Community assembly}

A species-rich community can never be realized by deleting species from a community (but see Tokita and Yasutomi 2003). Robinson and Valentine (1979) introduced the concepts of elasticity, invulnerability, and invadability. They randomly added a new species into a stable feasible equilibrium point of a system of linear differential equations of $n$ species and investigated stability of a new feasible equilibrium of the expanded system of $n+1$ species. They defined the original community as elastic, if the new equilibrium was stable and invasion of a new species was successful without accompanying extinction of other species. The original community was considered as invulnerable if the new equilibrium was unstable and the invader became extinct first. It was inelastic but vulnerable if the new equilibrium was unstable but species other than the invader went to extinction first. Thus, the invader was successful in invasion if the original community was elastic or inelastic but vulnerable. They found that stability of the original community and elasticity decrease and invulnerability increases as complexity increases. Yodzis (1981b) also examined the process of community assembly, assuming that sequentially arrived species can enter the existing community if the sum of acquired energy from randomly chosen species already present in the community exceeds the total required energy intake for the new arrival. Starting from a community of $N$ species of producers each with a productivity $P$ and assuming a fixed ecological efficiency $E$ of consumers, he could show that the assembled communities provide a reasonable model for 35 out of the 40 real food webs compiled by Briand (1983) on the basis of some web statistics. Post and Pimm (1983) also assembled model food webs by starting from six autotrophic species and successively introducing heterotrophic species. A colonizing species was rejected if it cannot increase when rare, if it completes a feeding loop, or if the expanded food web does not have a finite number of equilibria. When a colonist could invade, the other species with negative density at an equilibrium were deleted until a feasible food web was obtained. Finally, local stability of the equilibrium was checked and the potential colonist was rejected if it was unstable. They found that colonization becomes increasingly difficult with the number of attempted colonizations, that successional colonists do not cause serious multi-species extinctions, and that the return times of communities to equilibria increases with the increasing number of attempted colonizations. However, the mean number of species in communities stayed at the same order with the initial one.

\section{Scaling properties}

Since May's criterion implied the constraints on the product $S C$ for stable ecological communities, regularities in the product $S C$, the linkage density or the ratio of links to species numbers $(L / S)$, and the power-law relation between links and species numbers were sought (Cohen 1977a, 1978; MacDonald 1979; Rejmánek and Starý 1979; Briand 1983). The effort to find patterns in food-web network structure led to some scale-invariant patterns roughly independent of species numbers, such as the linkage density $L / S$ and the ratio of prey to predators (Briand and Cohen 1984; Cohen and Briand 1984; Lawton 1989; Cohen et al. 1990; Pimm et al. 1991; see Dunne 2006 for a recent review).

\section{Intervality and one dimensional niche}

Among patterns in food-web network structure, the patterns of which predators feed on which prey and those of which prey are fed on by which predators are quite important because the predators sharing common prey and the prey sharing common predators potentially compete exploitatively or apparently. Thus, graph-theoretical patterns 
of overlaps in resource (prey) uses among consumers (predators) and those in enemies (consumers or predators) among resources (prey) were extensively studied (Cohen 1977b, 1978; Sugihara 1982, 1984). A community food web is described as a directed graph consisting of vertices representing species and edges that connect two vertices by an arrow from a prey vertex to a predator vertex (Gallopin 1972; Cohen 1978). Two non-directed graphs can be obtained from the community food web by choosing either consumers or resources and connecting vertices that share common resources or consumers. The former is a niche overlap graph (Cohen 1977b, 1978) or predator overlap graph (Pimm et al. 1991) and called a consumer (overlap) graph by Sugihara $(1982,1984)$. The latter is a common enemy graph (Lundgren and Maybee 1985) or prey overlap graph (Pimm et al. 1991) and called a resource graph by Sugihara $(1982,1984)$. Cohen $(1977 b, 1978)$ defined a food web as an interval web if the overlaps in the niche overlap graph can be expressed as overlapping segments on a 1-dimensional line and found that food webs are more interval than expected by chance alone. Sugihara (1982, 1984) characterized similar properties of food webs by the rarity of topological holes (circuits connecting three or more prey species are simplexes or solids if the species share at least one common predator and called holes if there is no single predator common to all the prey species) with a dimension higher than one, and the high frequency of rigid circuits (food webs in which all the circuits with four or more predators are shortened or triangulated by edges across the circuits). The rigid circuit property often implies an interval representation of the niche overlap graph, but it does not guarantee that the food web is interval (Pimm et al. 1991). These regularities suggested that the dimension of trophic niche space can be one because the range of variation in the diet of each consumer can be identified with an interval on a line (Cohen 1977b, 1978; Cohen and Palka 1990). Being aware of the failure of static food web models constructed on the assumption that any species can eat any other species, Cohen and Newman (1985) invented a food web model with a strict trophic hierarchy. In the cascade model, a given species can prey on only those species below it and can be preyed on by only those species above it in the hierarchy. Cohen and Newman (1985) and Cohen et al. (1990) found that the cascade model can explain topological properties of food webs much better than the random model by tuning two parameters, species richness $S$ and link density $L / S$. Intervality and lack of holes are also related to the upper limit to the number of trophic interactions in a food web (Matsuda and Namba 1991). Recent development in studies of intervality will be found in Stouffer et al. $(2006,2011)$ and Zook et al. (2011).

\section{Intraguild predation}

The discovery of intraguild predation by Polis and his colleagues (Polis and McCormick 1986, 1987; Polis et al. 1989) was one of the important topics in the 1980s that influenced later research on relationships between complexity and stability. Intraguild predation is a kind of simple omnivory in which one of two consumers (intraguild predator) sharing a common resource feeds on the other (intraguild prey). The term "intraguild predation" appeared in 1986 (Polis and McCormick 1986) and became rapidly popular in a few years (Polis et al. 1989). It played an important role in later years to reveal the effects of interaction strengths on stability of food webs.

\section{Improved web data}

By the early 1990s, food web researchers found that food webs from a wide range of communities share a remarkable list of patterns (Lawton 1989; Cohen et al. 1990; Pimm et al. 1991), but at the same time they confronted with limited data of highly variable quality and were in serious quest of improved data collected by standardized explicit sampling methods reporting yield-effort curves and satisfying consistency of taxonomic identification (Lawton 1989; Pimm et al. 1991; Cohen et al. 1993). Then, an entirely new level of empirical food webs were provided by Polis (1991) and Martinez (1991) in 1991 (Dunne 2006). Other detailed but a little less complete food webs, for example, Warren (1989) and Winemiller (1990), were also published at about the same time (Dunne 2006).

\section{Models of food web structure}

The accumulation of highly resolved webs in the 1990s stimulated construction of food web structure models to explain topological properties found in the new webs. Williams and Martinez (2000) extended the cascade model by a little relaxing the hierarchical nature of trophic relations in the cascade model. The niche model by Williams and Martinez (2000) used two adjustable parameters, the number of species $S$ and directed connectance $C=L / S^{2}$ (Martinez 1991), and assigned each species a uniformly random niche value along an interval $[0,1]$ and a feeding range in the interval. A feeding range was centered at a randomly chosen place below the niche value of the species and the range size was chosen randomly from a beta distribution to obtain a connectance $C$ close to the target $C$. The niche model permitted feeding loops and cannibalism and successfully predicted structural properties of most complex food webs. However, the niche model can produce only interval food webs and entail no gap in diets 
for a suitable ordering of the prey. Cattin et al. (2004) considered that species' diets are the consequence of phylogenetic constraints and adaptation and proposed a new model, the nested hierarchy model. The model chooses the number of links per consumer reflecting the niche value and then trophic links are assigned to consumers starting with the smallest niche value. If a resource species randomly selected by a consumer is also fed upon by other consumers, a consumer group is defined and the next feeding link is randomly selected from the pool of resource species of the consumer group. Although Cattin et al. (2004) concluded that the nested hierarchy model performs as well as the niche model in the predictions of standard food-web descriptors and surpasses the niche model for two additional properties, there are unfavorable comments on their claims on selective favoring of intervality at the possible expense of reduced fit to other properties (Dunne 2006). For critical assessments of models of food web structure, refer to Stouffer et al. (2005) and Dunne (2006).

\section{Interaction strength}

In May's criterion for stability $\left(\sigma^{2} S C<1\right)$, effects of the interaction strengths $\sigma$ have still remained to be resolved. Empirical studies that have estimated per capita interaction strength have found distributions skewed toward many weak interactions and few strong interactions (Paine 1992; Berlow 1999; Berlow et al. 2004; Wootton and Emmerson 2005). Observed non-random patterning of strong and weak links has been suggested to be critical for stability or persistence of theoretical and empirically observed complex communities (de Ruiter et al. 1995; Neutel et al. 2002); long feedback loops in food webs contain relatively weak links (Neutel et al. 2002) and the strongest negative effects are restricted to short loops concentrated at the lower trophic positions (de Ruiter et al. 1995). Short loops including strong links are usually omnivorous loops and the maximum weight of omnivorous loops starting from bottom prey through intermediate predator to top predator and ending at basal prey is strongly related to food-web stability in soil food webs (Neutel et al. 2007). In a large Caribbean marine food web, strong interactions on two consecutive levels of food chains are infrequent unless they are accompanied by strong omnivory (Bascompte et al. 2005). A theoretical study on food chains with four species (Emmerson and Yearsley 2004) found that a skew towards weak interactions promotes local and global stability only when omnivory is present and that interaction strengths skewed toward weak interactions along omnivorous loops are an emergent property of stable omnivorous communities. These results strongly suggest that efficiency of indirect path from basal prey to top predator through intermediate predator is not only important for stability of an omnivorous loop (Tanabe and Namba 2005; Namba et al. 2008) but also crucial for community stability.

McCann and Hastings (1997) considered a dynamical model of intraguild predation including the type II functional responses of the top predator to the consumer and resource. They introduced a parameter which controls the top predators' preference for either the resource or consumer, and showed that weak predation of the top predator on the basal resource (omnivory) can stabilize food chains which exhibit chaos in the absence of omnivory. Using similar frameworks, McCann et al. (1998) introduced a second consumer to a linear food chain and then added links between the top predator and second intermediate consumer and between two intermediate consumers. The two added links created apparent competition and intraguild predation between two consumers, respectively. They showed that weak links can stabilize the chaotic food chain and produce well-bounded limit cycle solutions but failed to obtain locally stable equilibria. They attributed the reason to the existence of fewer inhibitors than oscillators. Finally they added a link between the top predator and resource into the food chain with a second consumer and succeeded to get a locally stable solution for weak relative interaction strengths. These results are often invoked as evidence to show that particular combinations of weak and strong interactions can stabilize food webs. However, since weak and strong interactions are introduced in pairs and a trade-off is assumed to weaken a strong interaction when a weak interaction is enhanced, combinations of weak and strong interactions are restricted in these studies. To reveal the role of diversity and a few strong links embedded in webs of many weak links, mathematical models of large food webs in which strong links are placed at specific positions should be studied.

\section{Biodiversity and ecosystem functioning}

In the 1990s, from increasing concern on loss of biodiversity, studies on the relationship between biodiversity and ecosystem functioning became a central issue in ecological and environmental sciences (Schulze and Mooney 1993; Loreau et al. 2001). These studies focused on the relations between biodiversity and many aspects of ecosystem properties and services including primary productivity, nutrient use and retention, and stability of these processes (Vitousek and Hooper 1993; Naeem et al. 1994; Tilman and Downing 1994). Long term studies of grasslands in Minnesota (Tilman 1996, 1999) and the panEuropean BIODEPTH experiment with standardized protocols (Hector et al. 1999) demonstrated that greater plant 
diversity leads to greater productivity. The sampling or selection effect hypothesis suggested that the positive effects are caused by the greater chance of including a few dominant, high performance species in a diverse plant community (Aarssen 1997; Huston 1997; Tilman et al. 1997; Loreau 2000). In contrast, the niche complementarity hypothesis assumed that more efficient use of limiting resources by niche differentiation among diverse species leads to higher productivity (Tilman et al. 1997; Loreau 1998, 2000).

Empirical studies showing that diversity enhances stability used resistance to disturbance and temporal variation in community biomass among many different measures of stability and invoked competitive interactions to understand why diversity promotes stability of aggregate community properties (McNaughton 1977; Tilman and Downing 1994; Tilman 1996, 1999). Tilman (1996) found that the coefficient of variation, a measure of the inverse of temporal stability, correlates with species richness significantly negatively for community biomass and positively for individual species biomass. This means that higher plant diversity is associated with greater stability of community biomass and lower stability of individual species biomass (Tilman 1996). He ascribed the results to different susceptibility of species to disturbance and compensatory growth of species released from competition with disturbance-susceptible species, referring to the hypothesis of compensatory interactions to environmental fluctuations among co-occurring species (McNaughton 1977). Tilman (1996) considered the complexity-stability relationship primarily based on May's model of competition (May 1973). In the model, all the eigenvalues of the community matrix have negative real parts but the dominant eigenvalue approaches zero as species richness increases. Tilman (1996) quoted the afterthoughts in the revised 1974 edition of May's book that suggest stability of aggregate properties of communities composed of unstable individual populations. For a mechanism of rising stability with increasing diversity in Tilman (1996), Doak et al. (1998) proposed the statistical averaging of fluctuations in species' abundances, which was called the portfolio effect by Tilman et al. (1998). Tilman et al. (1998) introduced the variance-mean rescaling and showed that the effect of diversity on community stability depends on the exponent, or the relationship between the mean and its variance. They also considered the negative covariance between variables which might be caused by interspecific competition and suggested the importance of investigating the relative contribution of the statistical averaging and interspecific competition (see Tilman 1999; Cottingham et al. 2001; and Tilman et al. 2014 for reviews).

\section{Metabolic theory of ecology and bio-energetic model}

In the late 1990s, "metabolic theory of ecology" based on allometric scaling of metabolic rates with body mass has experienced a renaissance (Brown et al. 2004; Sibly et al. 2012). Metabolism is the biochemical processes of uptake, transformation, and allocation of energy and materials within a living organism (Brown et al. 2004; Sibly et al. 2012). The metabolic rates are the fundamental biological rates and often described as power functions of body size (body mass) called allometric relations (Peters 1983). Studies of the relations have a long history (Peters 1983; Whitfield 2006) including the contribution by Huxley (1932) and the famous 3/4 (three quarters) law by Kleiber (1932). However, the meeting of a physicist, Geoffrey West, with two ecologists, Brian Enquist and James Brown at the Santa Fe Institute in 1995 ignited the enthusiastic search for the mechanisms and extensions to wider biological phenomena (Whitfield 2006).

Emmerson and Raffaelli (2004) estimated per capita interaction strengths between prey and predators (coefficients of the Lotka-Volterra model) from an allometric relation with the predator-prey size ratio and showed that the maximum real part of the eigenvalues of the community matrix is always negative and that the real complex food web is stable. Yodzis and Innes (1992) already estimated mass-specific metabolic rates through the allometric relations with the negative quarter power of body size, constructed the so-called bioenergetic model including nonlinear functional responses of predators (Brose et al. 2006), and analyzed the model. Otto et al. (2007) parametrized a bioenergetic model of food chains with three trophic levels. They found that almost all of tri-trophic food chains across five natural food webs exhibit body mass ratios between top and intermediate species and between intermediate and basal species within the persistence domain predicted from the dynamic model. By two types of random rewiring of food webs, the first preserving the body masses of the species and the total number of links and the second preserving the body mass and number of links for each species, they concluded that body mass and allometric degree distributions in natural food webs mediate the consistency.

Williams and Martinez (2004) and Martinez et al. (2006) generalized the bio-energetic model to $n$ species and arbitrary functional responses. They described the functional response in terms of the $(1+q)$-th power of body size $B$ and examined stability of the model for values of a control parameter $q$ between 0 and $1 \quad(q=0$ and 1 respectively corresponds to the type II and III functional response). Williams and Martinez (2004) examined dynamics of the three species food chain model studied by McCann and Hastings (1997) and found that a dramatic 
change occurred as $q$ increased. When $q=0$, the system with the type II functional response exhibited chaos, but the system was stabilized through period-doubling reversals as $q$ increased and finally reached a stable stationary solution when $q \approx 0.2$. They named the functional response corresponding to $q=0.2$ the "type II.2" response. Martinez et al. (2006) built model food webs of large size assuming the initial topological structure of the random, modified cascade, and niche webs (Williams and Martinez 2000). They suggested that the hierarchical ordering of the cascade model and the contiguous niches allowing cannibalism and looping in the niche model enhanced the persistence of populations and that both predator interference and type II.2 functional response showing respective decelerated and accelerated responses on rare and abundant resources had stabilizing effects even in large networks with many species. However, relative persistence, or the ratio of the final to initial number of species decreased linearly with the increasing initial network size and connectance, thus qualitatively replicating May's results (Dunne et al. 2005). Brose et al. (2006) analyzed a similar model with the initial structure following the modified cascade, niche or nested hierarchy model assuming that the mass-specific metabolic rates followed negative-quarter power law relationships with the predator-prey body mass ratios. In their simulations, most populations exhibited chaotic or limit cycle dynamics, and few populations reached equilibrium. They found that body mass ratios are the most important determinant of population and community stability and that food web stability (equivalent to the relative persistence in Martinez et al. 2006) consistently increases with the predator-prey body mass ratios. In their case, the relation between species richness and species' probability of persistence is negative when body size ratios are smaller than $10^{1}$ but positive at ratios of $10^{2}$ and above. Thus, their results partially reproduce and partially refute the classic result (May 1972). They suggested that average body mass ratios well above unity, usual in natural systems, yield neutral or positive diversity-stability relationships.

\section{Complex network theory}

In the 2000s, ecological networks including food webs and mutualistic networks were extensively studied from the perspectives of complex networks (Montoya et al. 2006). Complex networks are characterized by the average shortest distance between two nodes, the clustering coefficient, and the degree distribution or the distribution of the number of links per node (Watts and Strogatz 1998; Strogatz 2001). Based on these statistics, many real networks from a wide range of physical, biological, and social systems share the properties of the "small-world" with short distances between any two randomly chosen nodes and highly clustered and "scale-free" with degree distribution decaying as a power law (Watts and Strogatz 1998; Amaral et al. 2000; Strogatz 2001). Scale-free networks are characterized by robustness to errors, or random removals of nodes, and extreme vulnerability to attacks, or selective removals of highly connected nodes (Albert et al. 2000). However, most food webs do not have small-world and scale-free structure unless the connectance is relatively low (Dunne et al. 2002a), and many food webs and mutualistic networks have degree distributions described by functions such as exponential like or truncated power law with exponential decay (Camacho et al. 2002; Dunne et al. 2002a; Jordano et al. 2003; Stouffer et al. 2005; Montoya et al. 2006).

\section{Secondary cascading extinctions after a primary loss of species}

In the light of the complex network theory, sensitivity of food webs to primary species loss was investigated as the risk of secondary cascading extinctions from a little different view point from deletion stability (Pimm 1979b, 1980). Borrvall et al. (2000) considered food web models of three trophic levels with two to six species per trophic level. They assumed that average per capita effect of a consumer on its prey is inversely related to the number of prey species it consumes in the Lotka-Volterra model and examined the probability that the system has a locally stable feasible steady state after the removal of one species. They found that the risk of cascading extinctions decreases with the number of species per trophic level and that stability is lower when the distribution of interaction strengths is skew (few strong and many weak links). In contrast, Solé and Montoya (2001) and Dunne et al. (2002b) simulated secondary extinctions resulting from primary species loss in real food webs. Dunne et al. (2002b) found that food webs with less skewed degree distributions than the scalefree networks are also more robust to random removal of species than to selective removal of the most connected species. They also found that robustness, measured as the proportion of primary species removals that lead to loss of more than half species, increases with food web connectance but does not vary systematically with species richness or omnivory. Dunne and Williams (2009) compared levels of secondary extinctions in communities generated by four structural food-web models, the cascade model (Cohen and Newman 1985), the generalized cascade model (Stouffer et al. 2005), the niche model (Williams and Martinez 2000), and the nested hierarchy model (Cattin et al. 2004) and a fifth null model. They found that the null model displays the lowest secondary extinctions and suggested that hierarchical feeding assumed in structural 
models may impose a cost in terms of robustness. They also found that more realistic models with exponential-type degree distributions have greater structural robustness and that increased robustness is associated with increased species richness and connectance.

\section{Mutualistic networks}

Throughout the 1970s to the 1990s, ecologists were prone to be interested in networks composed of antagonistic interactions such as food webs or competitive communities. However, from the network approaches they learned the importance of studying patterns of interactions among species beyond predator-prey webs to include mutually beneficial interactions (Jordano et al. 2003) and benefited from tools and concepts imported from other fields such as physics and sociology (Bascompte 2009). Based on the findings that plant-animal mutualistic systems are not restricted to specialized one to one relations but include interactions between many plants and many animals (Waser et al. 1996), it was found that mutualistic networks are asymmetric and heterogeneous (Jordano 1987). Moreover, they are highly nested, that is, most species are specialists and interact only with proper subsets of species interacting with the more generalists (Bascompte et al. 2003; Bascompte and Jordano 2007).

Bascompte et al. (2006) defined the mutual dependence between each plant and animal species as the relative frequency of visits and found that the frequency distribution of dependencies is highly skewed with a few strong ones. The distributions are also asymmetric and strongly dependent animals (plants) on plants (animals) tend to be weakly depended by the plants (animals). They also considered the strength of an animal (plant) species, the sum of dependences of the plants (animals) relying on this animal (plant), a measure of the importance of this animal (plant) for plants (animals). They described dynamics of animals and plants by the Lotka-Volterra equations and assumed that per-capita effects of animals and plants in the equations can be estimated by the dependences. They showed that for a positive stable steady state to exist the product of mutual dependences must be smaller as the numbers of plant and animal species become larger. They suggested that the small number of strong dependences, their asymmetry, and the heterogeneity in species strengths promote community coexistence.

Okuyama and Holland (2008) developed a dynamic model of mutualistic communities with a hyperbolic functional response representing that the benefits to mutualists saturate with the densities of mutualistic partners with which they interact. Because this model always has a locally stable equilibrium, they evaluated community stability using resilience, return rate to the equilibrium. As a result, the model of mutualistic communities exhibited largely positive complexity-stability relationships and they attributed the positive relationships to the degree distribution described by the modified power law, nestedness of the mutualistic networks, community size, and the strength and symmetry of mutualistic interactions. These results seem contrary to the belief that the positive feedback of mutualism destabilizes communities and the results by Bascompte et al. (2006) that asymmetric interaction strengths may facilitate local stability of mutualistic communities. Symmetry of interaction strengths is defined as positive correlations between the half saturation constants of animals and plants in their model. However, interaction terms as a whole may be asymmetric because they contain partners' densities in denominators.

Bastolla et al. (2009) incorporated competition among animals and among plants to a similar model studied by Okuyama and Holland (2008). They evaluated effect of mutualism by the increase in the number of coexisting species due to mutualism. They showed that nestedness reduces the effective interspecific competition and enhances the number of coexisting species. They also found that if a new species entering the community interacts with the most generalist species, it will experience the lowest competitive load, and will therefore be most likely to be incorporated into the community. They incorporated the structure of each one of 56 real mutualistic networks into their model and found that the more nested real communities show the higher increases in biodiversity due to mutualism. In such models of mutualistic networks, benefits gained by plants from animal services such as pollination and seed dispersal are often assumed to be independent of rewards that animals receive. Since both the benefits to plants and rewards to animals may be limited by the number of animal visits to plants, models incorporating more realistic assumptions on saturation of benefits and competition for a limited number of mutualistic partners seem necessary.

\section{Adaptive flexible foraging}

In food webs, the "who eats whom" relations are determined by predator's choice of prey and may vary temporally (Warren 1989). Kondoh (2003a) introduced the idea of adaptive or flexible foraging into food web theory. $\mathrm{He}$ assumes that a consumer species increases (decreases) its foraging effort allocated to a resource species if energy gain per unit effort from the resource species is higher (lower) than the average profitability of resources that the consumer is currently foraging. He examined the dynamics of the foraging effort along with the population dynamics of the Lotka-Volterra type and found positive complexitystability relationship based on community persistence, the 
probability that all species persist for a given time as an index of stability. However, Brose et al. (2003) criticized three assumptions in Kondoh (2003a); the use of the random and cascade models, type I functional response, and the positive growth terms that allow consumers to grow without feeding on resources. Measuring stability by community robustness, the fraction of species within a network that persist, they found that the positive complexity-stability relationship appears in cascade model food webs only when initial connectance is higher than 0.2 and adaptation rate is 0.025 or higher. Furthermore, they could not find the positive relationship in niche model networks and concluded that positive complexity-stability relationships due to adaptive foraging are unlikely to be found for realistic parameter values in complex network structures (Brose et al. 2003; Dunne et al. 2005). Kondoh (2003b) challenged them on a "hidden treatment" of decreasing fraction of producer species and the emphasis on "potential connectance" instead of realized connectance. He considered that distribution of realized trophic links are biased by biomass distribution pattern and that this is likely to conflict the basic assumption of niche model. At any rate, adaptive flexible foraging contributes to persistence of populations by preventing rare species from being overexploited to extinction when asymptotic dynamics of networks exhibit heteroclinic cycles (Law and Morton 1993).

\section{Food webs including parasites}

Parasites have been rather neglected in food web studies (Huxham et al. 1995; Marcogliese and Cone 1997; Lafferty et al. 2006, 2008). However, the numbers and proportion of parasitic species are much higher than estimated before (Poulin and Morand 2000; Dobson et al. 2008) and parasites can contribute substantial biomass to estuarine ecosystems (Kuris et al. 2008). Incorporation of parasites sometimes dramatically alter properties of food webs. Besides obvious increases in species richness, number of links, linkage density, and food chain length (Huxham et al. 1995; Marcogliese and Cone 1997; Lafferty et al. 2006; Amundsen et al. 2009) parasites with complex life cycles increase proportion of omnivores because of feeding on several different trophic levels at different life stages (Lafferty et al. 2008; Amundsen et al. 2009). Furthermore, addition of parasites often increases connectance and nestedness (Lafferty et al. 2006; Amundsen et al. 2009) and increases link density and connectance particularly when including concomitant links from predators to parasites of their prey (Dunne et al. 2013). However, the addition of parasites into food webs increases secondary extinctions after loss of primary free-living species and reduces food web robustbess, because parasitic species with several specialized life stages act as sequential specialists hypersensitive to resource loss (Lafferty and Kuris 2009; Rudolf and Lafferty 2011). Degree distributions of food webs including parasites show exponential-type shapes similar to those observed for previously studied webs and are consistent with scale-dependent changes in structure, but the trophic niches of parasites tend to be broader and have more gaps (Dunne et al. 2013). Inclusion of concomitant links substantially changes distributions of threenode motifs or patterns of interactions among three species. Bidirectional interactions made up of one parasite-host interaction and one concomitant link appear far more frequently in some motifs than in others (Dunne et al. 2013). It should be clearly assessed whether such changes in network structure result from unique roles and traits of parasites or merely from changes to diversity and complexity (Chen et al. 2011; Dunne et al. 2013).

\section{Frontiers in the 2010s}

Following interests in many types of ecological networks, comparing architecture among different network types and merging various interaction types within a single integrative network became a challenge necessary to understand the structure and dynamics of ecological communities (Ings et al. 2009; Fontaine et al. 2011). In theoretical studies, based on development in mathematical backgrounds of May's original theory, new extension to May's conclusion is sought for and new application of statistical mechanics to nonlinear dynamics is also tried. Rapid innovation in molecular techniques enables us to see 'the unseen majority' (e.g., soil microbes; van der Heijden et al. 2008) and empirical studies on complex networks are celebrating a new era. In this section, I introduce these new movements, mainly focusing on works by the authors of this Special Feature.

\section{Complexity and stability in communities with multiple trophic levels}

Thébault and Loreau (2003) examined effects of biodiversity in a model of food webs incorporating multiple trophic levels and plant competition for nutrient. They found that multiple trophic levels produce complex relationships between species richness and ecosystem processes different from those within a single trophic level. In chains of plants and herbivores, total plant biomass did not increase linearly but initially increased and then decreased with diversity of plants and herbivores when a plant species was inedible or herbivores were generalists. In chains including carnivores, a decrease in mean total plant biomass at high diversity appeared when a trade-off between competitive ability and resistance to herbivory was assumed 
because herbivores were controlled by carnivores and biomass of the more tolerant and less competitive plants decreased due to reduced soil nutrient concentration. Thébault and Loreau (2005) incorporated responses of plants and herbivores to environmental fluctuations into a similar model. Then, variability of species biomass often increased but that of trophic level biomass usually decreased. Thus, in spite of the complexity generated by trophic interactions, biodiversity acted as biological insurance for ecosystem processes against environmental fluctuations. However, differing from purely competitive systems, the temporal variability of total plant and herbivore biomasses could still decrease in webs of plants and herbivores even if species responded identically to environmental changes.

\section{Networks including mutualistic and antagonistic interactions}

Melián et al. (2009) studied an ecological network including mutualistic and antagonistic interactions at a biological station in southern Spain. They considered modules defined by a plant species interacting with a pollinator or seed disperser and an herbivore. Their finding that the number of the modules is larger than expected by chance suggested that a plant species having an antagonistic interaction tends to have a mutualistic interaction more than expected by chance. The frequency distribution of the number of modules per plant species was quite heterogeneous and best described by a power law. By the analysis of a simple dynamic model, they found that the fraction of persistent plant species is significantly higher when the observed network topology and the correlation between the ratio of mutualistic to antagonistic interactions per plant and interaction strength (sum of dependences of animal species on the plant species) are incorporated.

Network architecture favoring stability fundamentally differs between trophic and mutualistic networks (Thébault and Fontaine 2010). For both plant-pollinator and plantherbivore networks asymmetric specialization in terms of the number of links per species is significant but most of the pollination networks are highly asymmetric and most of the herbivory networks are weakly asymmetric (Thébault and Fontaine 2008). Pollinators interact with more plant species and more evenly than herbivores but herbivores interact with plant species far more phylogenetically related to each other (Fontaine et al. 2009). Building a population dynamics model, Thébault and Fontaine (2010) found that higher diversity and connectance promote persistence and resilience of mutualistic networks but destabilize trophic networks. Nestedness and modularity also have different effects on persistence and resilience of mutualistic and trophic networks; nestedness increases resilience of mutualistic networks and decreases persistence of trophic networks; in contrast, modularity decreases persistence of mutualistic networks and enhances resilience of trophic networks.

In this Special Feature, Fontaine and Thébault (2015) compared conservatism, the tendency that related species interact with similar partners, between mutualistic and antagonistic networks. The positive correlation between the taxonomic relatedness and the overlap in interacting partners was found the highest for plants interacting with herbivores, followed by pollinating insects, plants interacting with pollinators and by herbivorous insects. Their results suggest that within antagonistic networks, conservatism is stronger for resource species than for consumer species but not in mutualistic networks, and the difference in conservatism between the pollinators and the plants they interact with is much smaller.

\section{Effects of interaction-type mixing}

Mougi and Kondoh (2012) proposed a hybrid community model in which both the antagonistic and mutualistic interactions are incorporated. They constructed model communities for random, cascade, and bipartite networks assuming Holling's type I or type II functional responses and a negative relationship between the number of interactions and the interaction strength. They showed that a small number of mutualistic interactions added to a locally stable food web strongly destabilize the community but that by a further increase in the proportion of mutualism the community becomes more stable and reaches its peak stability at an intermediate proportion of mutualistic links. They also found the positive complexity-stability relationship and considered that the interaction type-mixing and negative relation between the number and strength of interactions are the key assumptions. However, Suweis et al. $(2013,2014)$ claimed that the positive complexitystability relationship observed in Mougi and Kondoh (2012) is the result of two key assumptions; (1) constant efforts allocated separately to antagonistic and to mutualistic interactions independent of the total number of species engaged in either interaction types and (2) total interaction strengths spent in mutualistic (antagonistic) interactions independent of the proportion of mutualistic (antagonistic) links.

In this Special Feature, Kondoh and Mougi (2015) modified the model in Mougi and Kondoh (2012). They considered two types of effort allocation to interactions; (1) separate effort allocation, or constant effort allocation to each of two interaction types, and (2) mixed effort allocation, or constant total effort allocation to two interaction types. They found that the positive complexity-stability relationship appears when the strengths of the two 
interaction types are well-balanced and that stability maximum appears at the highest connectance even if all the effort allocation is of the mixed type. They also observed maximum community stability at intermediate proportion of mutualistic links and confirmed positive effects of interaction-type mixture. Recently Sauve et al. (2014) investigated effects of mixing interaction types on persistence, the proportion of species persisting at the end of the simulation, and resilience of networks by combining separately constructed bipartite antagonistic and mutualistic subnetworks. They found that the greater initial antagonistic (mutualistic) diversity and connectance decreases (increases) the persistence of the merged network and that the greater final antagonistic (mutualistic) diversity and connectance lower (enhance) the resilience. Thus, further studies seem necessary to fully understand the effect of interaction-type mixing.

\section{Extension of May' criterion}

The essence of May's theory in 1972 is built on analysis of eigenvalues of large random matrices based on Wigner's semicircle law. Although May's matrices contained fixed proportions of three types of interactions, consumer-resource (predator-prey), mutualism, and competition, Allesina and Tang (2012) extended May's criterion to separate networks of consumer-resource, mutualistic, and competitive interactions and then considered mixed ones composed of three types of interactions at arbitrary proportions. Based on the development of random matrix theory (Sommers et al. 1988; Tao et al. 2010), they analytically obtained distributions of eigenvalues enclosed in circles or vertically or horizontally stretched ellipses. In their results, the predator-prey matrices are the most likely to be stable and the competitive matrices are much less likely to be stable followed by the mutualistic matrices that are further slightly less likely to be stable. They also obtained several stability properties contrary to current beliefs; the cascade and niche models which impose realistic structure to food webs produce less stable networks. In mutualistic networks, nested matrices are inherently less likely to be stable. Weak interactions destabilize food webs but stabilize mutualistic and competitive networks. These results suggest the necessity to examine which components of complex networks contribute to stability in combination with weak interactions.

Tang et al. (2014) examined effects of several properties on stability of food webs. They estimated elements of community matrices for real food webs assuming allometric scaling between body size and the search rate, handling time, and equilibrium abundance. By five randomization tests each of which preserves some of the features in the empirical community matrix they found that the negative correlation between the effects of consumers on resources and those of resources on consumers greatly affects stability of real food webs. This correlation is strongly influenced by the scaling of species abundance with body size and the scaling exponent close to $-3 / 4$ maximizes the strength of correlation. Conserving the pairwise correlation produces matrices that are often more likely to be stable than the corresponding empirical one but preserving the topological network structure has little effect on stability (Tang et al. 2014). This study clearly shows the importance of equilibrium species abundance in calculating elements of a community matrix.

In this Special Feature, Allesina and Tang (2015) showed how to derive May's stability criterion using random matrix theory, and then explained how we can apply the so-called circular or elliptic law for the distribution of the eigenvalues of a large random matrix to investigate stability of various ecological networks, step by step in an accessible way to ecologists. Then, they proposed three suggestive research programs. The first challenge is concerned with whether non-equilibrium persistence of large ecological networks can be predicted by the methods developed for local stability. The second is the effect of species-abundance distribution on stability of communities. When elements of an interaction matrix and equilibrium abundances are sampled independently eigenvalues of the community matrix are distorted. Then, the elliptic law fails to be applied and a new tool is suggested for resolving the challenge. The final challenge can be divided into three parts; sparse interactions, modularity or the existence of species groups, and network structure. Solving these challenges will contribute to understandings of ecological networks beyond the perspective of the complexity-stability relationship.

\section{Replicator equations and approaches from statistical mechanics}

Natural systems are not necessarily resting at equilibrium but often functioning out of equilibrium. Simulating nonlinear bioenergetics models incorporating increasingly realistic assumptions may be a solution to understand persistence of communities achieved through more complex dynamics, such as limit cycles or chaos (Brose et al. 2006; Heckmann et al. 2012; Kalinkat et al. 2013). However, these models are analytically intractable.

Tokita and his colleagues used replicator equations (Hofbauer and Sigmund 1988), a trajectory of whose solution is topologically equivalent to one of the corresponding Lotka-Volterra equations. They applied some techniques of non-equilibrium statistical physics to analyze global behavior of the system with random interactions (Tokita 2004, 2006; Yoshino et al. 2007, 2008). In this 
Special Feature, Tokita (2015) reviewed the history and recent progress of the analytical theories of random community models and species abundance distributions based on statistical mechanics. He gave an overview of three types of distinct analyses for replicator equations with symmetric, antisymmetric and asymmetric random interactions. When interactions are symmetric, the average fitness increases with time and the dynamics converges to one of the fixed points depending on the initial condition. The rank abundance relation takes the form of a straight line like the geometric series or sigmoid curves on a logarithmic vertical axis and the transition occurs when 'productivity' shifts from low to high values. When interactions are fully antisymmetric, the set of surviving species is uniquely determined but the corresponding fixed point is linearly neutrally stable. The hyperplane on which densities of not-persisting species are zero is filled with neutrally stable orbits and population densities fluctuate. A striking feature in this case is that species as many as half of the initial species number can survive. When interactions are asymmetric, the replicator equations have a globally stable fixed point for productivity greater than a threshold. Such a fixed point becomes unstable for productivity less than the threshold and nonequilibrium behavior such as chaos or heteroclinic cycles appear. The threshold increases linearly as the degree of symmetry increases from antisymmetry to symmetry. In contrast, diversity monotonically decreases with the degree of symmetry and is the highest for antisymmetric interactions and the lowest for symmetric interactions. Symmetry and antisymmetry in the replicator equations do not mean the same in the Lotka-Volterra equations and a rich array of interactions including mutualism, competition, predation and parasitism appear in the corresponding Lotka-Volterra equations (Tokita and Yasutomi 2003).

\section{Revolution in sampling and processing of high throughput data}

We have very little data on networks including multiple types of interactions and data on various networks are not usually equitable (Fontaine et al. 2011). Ecological networks in the soil are far less understood than other networks due to the difficulty of sampling microbes and characterizing interactions and processes including fungi and bacteria (van der Heijden et al. 2008; Fontaine et al. 2011; Digel et al. 2014).

Toju and his colleagues overcame the difficulty by using the high-throughput DNA barcoding of microbes and subsequent appropriate processing of next-generation sequencing data and applied the method to reveal the architecture of ecological networks of co-occurring plant species and their root-associated fungi (Toju et al. 2013a, b, c, 2014a, b). In an oak-dominated temperate forest, ectomycorrhizal and root-endophytic fungi constituted a complex community and a number of endophytic fungi were shared by a broad range of host plant species (Toju et al. 2013a). However, the five most common species had specificity in their association with fungal taxa and fungi also displayed remarkable variation in their association specificity for plants (Toju et al. 2013b). Although an ectomycorrhizal fungus and a possible endophytic fungus significantly favored the dominant oak species and arbuscular mycorrhizal fungi were generally shared among subordinate plant species, diverse clades of ectomycorrhizal and possible root endophytes were associated not only with the dominant oak species but also with the remaining plant species (Toju et al. 2013c). Also in subtropical forests, belowground plant-fungal symbiosis included "non-typical" combinations and different groups of fungi (ectomycorrhizal and arbuscular mycorrhizal fungi) were differently shared among plant species (Toju et al. 2014a). Overall, the plant-fungus network was more compartmentalized than expected by chance but the network modularity was lower than that usually observed in plant-pollinator networks (Toju et al. 2014b). Although nestedness is often observed in ecological networks and considered to promote stable species coexistence in mutualistic networks (Bascompte et al. 2003; Thébault and Fontaine 2010), interactions in plant-fungus networks were not found nested and the nestedness was even lower than expected by chance (Toju et al. 2014b).

In this Special Feature, Toju (2015) reviewed the methodological platforms of next-generation sequencingbased analyses of microbe-host animal/plant networks in detail and introduced some studies on the plant-fungal networks by him and his colleagues. This review will definitely help the entry of many ecologists into this field. Since the technology substantially reduces the time cost of field sampling, it will promote comparative studies of belowground ecological networks and even make it possible to track the temporal dynamics of network architecture (Toju 2015).

\section{Coda}

In spite of the long history and multi-faceted approaches, there may still remain some problems expected to be solved by the time May's article turns 50 (Allesina and Tang 2015). Unfortunately, non-equilibrium dynamics of large complex ecological networks cannot be predicted from linear theory. Understanding precise dynamics of important community modules may help to fill the gap between the linear theory and computer simulations of models of communities with many species, since some community modules or motifs may play an indispensable 
role to determine community structure and dynamics (Bascompte et al. 2005; Kondoh 2008). Merging various interaction types within a single integrative network (Kéfi et al. 2012) will remain to be important for a next few decades. However, not phenomenological but mechanistic functional responses including interference between predators and between mutualists are necessary to construct hybrid models including multiple types of interactions. Finally, it is needed to establish a method to get essential information from a vast amount of high throughput barcoding data and draw a new picture of network structure including multiple interaction types, which may be highly different from the ones we know now. Then, collaboration between empirical and theoretical researchers will develop further more. I hope that this Special Feature can make some contribution to resolve the relationship between complexity and stability.

Acknowledgments This work was supported by JSPS KAKENHI Grant Number 26440247.

Conflict of interest The author declares that there are no conflicts of interest.

Open Access This article is distributed under the terms of the Creative Commons Attribution License which permits any use, distribution, and reproduction in any medium, provided the original author(s) and the source are credited.

\section{References}

Aarssen LW (1997) High productivity in grassland ecosystem: effected by species or productive species? Oikos 80:183-184

Albert R, Jeong H, Barabási A-L (2000) Error and attack tolerance of complex networks. Nature 406:378-382

Allesina S, Tang S (2012) Stability criteria for complex ecosystems. Nature 483:205-208

Allesina S, Tang S (2015) The stability-complexity relationship at age 40: a random matrix perspective. Popul Ecol. doi:10.1007/ s10144-014-0471-0

Amaral LAN, Scala A, Barthélémy M, Stanley HE (2000) Classes of small-world networks. Proc Natl Acad Sci USA 97:11149-11152

Amundsen P-A, Lafferty KD, Knudsen R, Primicerio R, Klemetsen A, Kuris AM (2009) Food web topology and parasites in the pelagic zone of a subarctic lake. J Anim Ecol 78:563-572

Bascompte J (2009) Disentangling the web of life. Science 325:416-419

Bascompte J, Jordano P (2007) Plant-animal mutualistic networks: the architecture of biodiversity. Annu Rev Ecol Evol Syst 38:567-593

Bascompte J, Jordano P, Melián CJ, Olesen JM (2003) The nested assembly of plant-animal mutualistic networks. Proc Natl Acad Sci USA 100:9383-9387

Bascompte J, Melián CJ, Sala E (2005) Interaction strength combinations and the overfishing of a marine food web. Proc Natl Acad Sci USA 102:5443-5447

Bascompte J, Jordano P, Olesen JM (2006) Asymmetric coevolutionary networks facilitate biodiversity maintenance. Science $312: 431-433$
Bastolla U, Fortuna MA, Pascual-García A, Ferrera A, Luque B, Bascompte J (2009) The architecture of mutualistic networks minimizes competition and increases biodiversity. Nature 458:1018-1020

Berlow EL (1999) Strong effects of weak interactions in ecological communities. Nature 398:330-334

Berlow EL, Neutel A-M, Cohen JE, de Ruiter PC, Ebenman B, Emmerson M, Fox JW, Jansen VAA, Jones JI, Kokkoris GD, Logofet DO, McKane AJ, Montoya JM, Petchey O (2004) Interaction strengths in food webs: issues and opportunities. J Anim Ecol 73:585-598

Borrvall C, Ebenman B, Jonssonn T (2000) Biodivesity lessens the risk of cascading extinction in model food webs. Ecol Lett 3:131-136

Briand F (1983) Environmental control of food web structure. Ecology 64:253-263

Briand F, Cohen JE (1984) Community food webs have scaleinvariant structure. Nature 307:264-267

Brose U, Williams RJ, Martinez ND (2003) Comment on "foraging adaptation and the relationship between food-web complexity and stability". Science 301:918

Brose U, Williams RJ, Martinez ND (2006) Allometric scaling enhances stability in complex food webs. Ecol Lett 9:1228-1236

Brown JH, Gillooly JF, Allen AP, Savage VM, West GB (2004) Toward a metabolic theory of ecology. Ecology 85:1771-1789

Camacho J, Guimerà R, Amaral LAN (2002) Robust patterns in food web structure. Phys Rev Lett 88:228102

Cattin M-F, Bersier L-F, Banašek-Richter C, Baltensperger R, Gabriel J-P (2004) Phylogenetic constraints and adaptation explain foodweb structure. Nature 427:835-839

Chen H-W, Shao K-T, Liu CW-J, Lin W-H, Liu W-C (2011) The reduction of food web robustness by parasitism: fact and artefact. Int J Parasitol 41:627-634

Cody ML, Diamond JM (eds) (1975) Ecology and evolution of communities. Belknap Press, Cambridge

Cohen JE (1977a) Ratio of prey to predators in community food webs. Nature 270:165-167

Cohen JE (1977b) Food webs and the dimensionality of trophic niche space. Proc Natl Acad Sci USA 74:4533-4563

Cohen JE (1978) Food webs and niche space. Princeton Univ Press, Princeton

Cohen JE, Briand F (1984) Trophic links of community food webs. Proc Natl Acad Sci USA 81:4105-4109

Cohen JE, Newman CM (1985) A stochastic theory of community food webs. I. Models and aggregated data. Proc R Soc Lond B 324:421-448

Cohen JE, Palka ZJ (1990) A stochastic theory of community food webs. V. Intervality and triangulation in the trophic-niche overlap graph. Am Nat 135:435-463

Cohen JE, Briand F, Newmann CM (1990) Community food webs: data and theory. Biomathematics, vol 20. Springer-Verlag, Berlin

Cohen JE, Beaver RA, Cousins SH, De Angelis DL, Goldwasser L, Heong KL, Holt RD, Kohn AJ, Lawton JH, Martinez N, O'Malley R, Page LM, Patten BC, Pimm SL, Polis GA, Rejmánek M, Schoener TW, Schoenly K, Sprules WG, Teal JM, Ulanowicz RE, Warren PH, Wilbur HM, Yodzis P (1993) Improving food webs. Ecology 74:252-258

Connor EF, Simberloff D (1979) The assembly of species communities: chance or competition? Ecology 60:1132-1140

Cottingham KL, Brown BL, Lennon JT (2001) Biodiversity may regulate the temporal variability of ecological systems. Ecol Lett 4:72-85

De Angelis DL (1975) Stability and connectance in food web models. Ecology 56:238-243 
de Ruiter PC, Neutel A-M, Moore JC (1995) Energetics, patterns of interaction strengths, and stability in real ecosystems. Science 269:1257-1260

Digel C, Curtsdotter A, Riede J, Klarner B, Brose U (2014) Unravelling the complex structure of forest soil food webs: higher omnivory and more trophic levels. Oikos 123:1157-1172

Doak DF, Bigger D, Harding EK, Marvier MA, O'Thompson D (1998) The statistical inevitability of stability-diversity relationships in community ecology. Am Nat 151:264-276

Dobson A, Lafferty KD, Kuris AM, Hechinger RF, Jetz W (2008) Homage to Linnaeus: how many parasites? Proc Natl Acad Sci USA 105:11482-11489

Dunne JA (2006) The network structure of food webs. In: Pascual M, Dunne JA (eds) Ecological networks: linking structure to dynamics in food webs. Oxford Univ Press, Oxford, pp 27-86

Dunne JA, Williams RJ (2009) Cascading extinctions and community collapse in model food webs. Phil Trans R Soc B 364:1711-1723

Dunne JA, Williams RJ, Martinez ND (2002a) Food-web structure and network theory: the role of connectance and size. Proc Natl Acad Sci USA 99:12917-12922

Dunne JA, Williams RJ, Martinez ND (2002b) Network structure and biodiversity loss in food webs: robustness increases with connectance. Ecol Lett 5:558-567

Dunne JA, Brose U, Williams RJ, Martinez ND (2005) Modelling food-web dynamics: complexity-stability implications. In: Belgrano A, Scharler UM, Dunne J, Ulanowicz RE (eds) Aquatic food webs: an ecosystem approach. Oxford Univ Press, Oxford, pp 117-129

Dunne JA, Lafferty KD, Dobson AP, Hechinger RF, Kuris AM, Martinez ND, McLaughlin JP, Mouritsen KN, Poulin R, Reise K, Stouffer DR, Thieltges DW, Williams RJ, Zander CD (2013) Parasites affect food web structure primarily through increased diversity and complexity. PLoS Biol 11:e1001579

Elton CS (1958) The ecology of invasions by animals and plants. Methuen, London

Emmerson MC, Raffaelli D (2004) Predator-prey body size, interaction strength and the stability of a real food web. J Anim Ecol 73:399-409

Emmerson M, Yearsley JM (2004) Weak interactions, omnivory and emergent food-web properties. Proc R Soc Lond B 271:397-405

Fontaine C, Thébault E (2015) Comparing the conservatism of ecological interactions in plant-pollinator and plant-herbivore networks. Popul Ecol. doi:10.1007/s10144-014-0473-y

Fontaine C, Thébault E, Dajoz I (2009) Are insect pollinators more generalist than insect herbivores? Proc R Soc B 276:3027-3033

Fontaine C, Guimarães PR Jr, Kéfi S, Loeuille N, Memmott J, van der Putten WH, van Veen FJF, Thébault E (2011) The ecological and evolutionary implications of merging different types of networks. Ecol Lett 14:1170-1181

Gallopin GC (1972) Structural properties of food webs. In: Patten BC (ed) Systems analysis and simulation in ecology, vol 2. Academic Press, New York, pp 241-282

Gardner MR, Ashby WR (1970) Connectance of large, dynamical (cybernetic) systems: critical values for stability. Nature 228:784

Gilpin ME (1975) Stability of feasible predator-prey systems. Nature 254:137-139

Gilpin ME (1979) Spiral chaos in a predator-prey model. Am Nat 113:306-308

Goodman D (1975) The theory of diversity-stability relationships in ecology. Q Rev Biol 50:237-266

Heckmann L, Drossel B, Brose U, Guill C (2012) Interactive effects of body-size structure and adaptive foraging on food-web stability. Ecol Lett 15:243-250

Hector A, Schmid B, Beierkuhnlein C, Caldeira MC, Diemer M, Dimitrakopoulos PG, Finn JA, Freitas H, Giller PS, Good J, Harris R, Hógberg P, Huss-Danell K, Joshi J, Jumpponen A,
Körner C, Leadley PW, Loreau M, Minns A, Mulder CPH, O’Donovan G, Otway SJ, Pereira JS, Prinz A, Read DJ, SchererLorenzen M, Schulze E-D, Siamantziouras A-SD, Spehn EM, Terry AC, Troumbis AY, Woodward FI, Yachi S, Lawton JH (1999) Plant diversity and productivity experiments in European grasslands. Science 286:1123-1127

Hofbauer J, Sigmund K (1988) The theory of evolution and dynamical systems: mathematical aspects of selection. Cambridge Univ Press, Cambridge

Holt RD (1977) Predation, apparent competition, and the structure of prey communities. Theor Popul Biol 12:197-229

Hsu SB, Hubbell SP, Waltman P (1978) A contribution to the theory of competing predators. Ecol Monogr 48:337-349

Huston MA (1997) Hidden treatments in ecological experiments: reevaluating the ecosystem function of biodiversity. Oecologia 110:449-460

Huxham M, Raffaelli D, Pike A (1995) Parasites and food web structure. J Anim Ecol 64:168-176

Huxley JS (1932) Problems of relative growth. Methuen, London

Ings TC, Montoya JM, Bascompte J, Blüthgen N, Brown L, Dormann $\mathrm{CF}$, Edwards F, Figueroa D, Jacob U, Jones JI, Lauridsen RB, Ledger ME, Lewis HM, Olesen JM, van Veen FFF, Warren PH, Woodward G (2009) Ecological networks-beyond food webs. J Anim Ecol 78:253-269

Jordano P (1987) Patterns of mutualistic interactions in pollination and seed dispersal: connectance, dependence asymmetries, and coevolution. Am Nat 129:657-677

Jordano P, Bascompte J, Olesen JM (2003) Invariant properties in coevolutionary networks of plant-animal interactions. Ecol Lett 6:69-81

Kalinkat G, Schneider FD, Digel C, Guill C, Rall BC, Brose U (2013) Body masses, functional responses and predator-prey stability. Ecol Lett 16:1126-1134

Kéfi S, Berlow EL, Wieters EA, Navarrete SA, Petchey OL, Wood SA, Boit A, Joppa LN, Lafferty KD, Williams RJ, Martinez ND, Menge BA, Blanchette CA, Iles AC, Brose U (2012) More than a meal... integrating non-feeding interactions into food webs. Ecol Lett 15:291-300

Kleiber M (1932) Body size and metabolism. Hilgardia 6:315-332

Kondoh M (2003a) Foraging adaptation and the relationship between food-web complexity and stability. Science 299:1388-1391

Kondoh M (2003b) Response to comment on "foraging adaptation and the relationship between food-web complexity and stability". Science 301:918

Kondoh M (2008) Building trophic modules into a persistent food web. Proc Natl Acad Sci USA 105:16631-16635

Kondoh M, Mougi A (2015) Interaction-type diversity hypothesis and interaction strength: the condition for the positive complexitystability effect to arise. Popul Ecol. doi:10.1007/s10144-0140475-9

Kuris AM, Hechinger RF, Shaw JC, Whitney KL, Aguirre-Macedo L, Boch CA, Dobson AP, Dunham EJ, Fredensborg BL, Huspeni TC, Lorda J, Manabe L, Mancini FT, Mora AB, Pickering M, Talhouk NL, Torchin ME, Lafferty KD (2008) Ecosystem energetic implications of parasite and free-living biomass in three estuaries. Nature 454:515-518

Lafferty KD, Kuris AM (2009) Parasites reduce food web robustness because they are sensitive to secondary extinction as illustrated by an invasive estuarine snail. Phil Trans R Soc B 364:1659-1663

Lafferty KD, Dobson AP, Kuris AM (2006) Parasite dominate food web links. Proc Natl Acad Sci USA 103:11211-11216

Lafferty KD, Allesina S, Arim M, Briggs CJ, De Leo G, Dobson AP, Dunne JA, Johnson PTJ, Kuris AM, Marcogliese DJ, Martinez ND, Memmott J, Marquet PA, McLaughlin JP, Mordecai EA, Pascual M, Poulin R, Thieltges DW (2008) Parasites in food webs: the ultimate missing links. Ecol Lett 11:533-546 
Law R, Morton RD (1993) Alternative permanent states of ecological communities. Ecology 74:1347-1361

Lawlor LR (1978) A comment on randomly constructed model ecosystems. Am Nat 112:445-447

Lawton JH (1989) Food webs. In: Cherrett JM (ed) Ecological concepts. Blackwell Scientific, Oxford, pp 43-78

León JA, Tumpson DB (1975) Competition between two species for two complementary or substitutable resources. J Theor Biol 50:185-201

Loreau M (1998) Biodiversity and ecosystem functioning: a mechanistic model. Proc Natl Acad Sci USA 95:5632-5636

Loreau M (2000) Biodiversity and ecosystem functioning: recent theoretical advances. Oikos 91:3-17

Loreau M, Naeem S, Inchausti P, Bengtsson J, Grime JP, Hector A, Hooper DU, Huston MA, Raffaelli D, Schmid B, Tilman D, Wardle DA (2001) Biodiversity and ecosystem functioning: current knowledge and future challenges. Science 294:804-808

Lundgren JR, Maybee JS (1985) Food webs with interval competition graph. In: Harary F, Maybee JS (eds) Graph and applications: proceedings of the first Colorado symposium on graph theory. John Wiley and Sons, New York, pp 245-256

MacArthur RH (1955) Fluctuations of animal populations and a measure of community stability. Ecology 36:533-536

MacArthur RH (1972) Geographical ecology: patterns in the distribution of species. Harper and Row, New York

MacDonald N (1979) Simple aspects of foodweb complexity. J Theor Biol 80:577-588

Marcogliese DJ, Cone DK (1997) Food webs: a plea for parasites. Trends Ecol Evol 12:320-325

Martinez ND (1991) Artifacts or attributes? Effects of resolution on the Little Rock Lake food web. Ecol Monogr 61:367-392

Martinez ND, Williams RJ, Dunne JA (2006) Diversity, complexity, and persistence in large model ecosystems. In: Pascual M, Dunne JA (eds) Ecological networks: linking structure to dynamics in food webs. Oxford Univ Press, Oxford, pp 163-185

Matsuda H, Namba T (1991) Food web graph of a coevolutionarily stable community. Ecology 72:267-276

May RM (1972) Will a large complex system be stable? Nature 238:413-414

May RM (1973) Stability and complexity in model ecosystems. Princeton Univ Press, Princeton

May RM, Leonard WJ (1975) Nonlinear aspects of competition between three species. SIAM J Appl Math 29:243-253

McCann KS (2000) The diversity-stability debate. Nature 405:228-233

McCann KS, Hastings A (1997) Re-evaluating the omnivory-stability relationship in food webs. Proc R Soc Lond B 264:1249-1254

McCann KS, Hastings AG, Huxel R (1998) Weak trophic interactions and the balance of nature. Nature 395:794-798

McMurtrie RE (1975) Determinants of stability of large randomly constructed systems. J Theor Biol 50:1-11

McNaughton SJ (1977) Diversity and stability of ecological communities: a comment on the role of empiricism in ecology. Am Nat 111:515-525

Melián CJ, Bascompte J, Jordano P, Křivan V (2009) Diversity in a complex ecological network with two interaction types. Oikos 118:122-130

Montoya JM, Pimm SL, Solé RV (2006) Ecological networks and their fragility. Nature 442:259-264

Mougi A, Kondoh M (2012) Diversity of interaction types and ecological community stability. Science 337:349-351

Naeem S, Thompson LJ, Lawler SP, Lawton JH, Woodfin RM (1994) Declining biodiversity can alter the performance of ecosystems. Nature 368:734-737

Namba T, Tanabe K, Maeda N (2008) Omnivory and stability of food webs. Ecol Complex 5:73-85
Neutel A-M, Heesterbeek JAP, de Ruiter PC (2002) Stability in real food webs: weak links in long loops. Science 296:1120-1123

Neutel A-M, Heesterbeek JAP, van de Koppel J, Hoenderboom G, Vos A, Kaldeway C, Berendse F, de Ruiter PC (2007) Reconciling complexity with stability in naturally assembling food webs. Nature 449:599-602

Okuyama T, Holland JN (2008) Network structural properties mediate the stability of mutualistic communities. Ecol Lett 11:208-216

Otto SB, Rall BC, Brose U (2007) Allometric degree distributions facilitate food-web stability. Nature 450:1226-1229

Paine RT (1966) Food web complexity and species diversity. Am Nat 100:65-75

Paine RT (1969) A note on trophic complexity and community stability. Am Nat 103:91-93

Paine RT (1980) Food webs: linkage, interaction strength and community infrastructure. J Anim Ecol 49:667-685

Paine RT (1992) Food web analysis through field measurement of per capita interaction strength. Nature 355:73-75

Peters RH (1983) The ecological implications of body size. Cambridge Univ Press, Cambridge

Pimm SL (1979a) The structure of food webs. Theor Popul Biol $16: 144-158$

Pimm SL (1979b) Complexity and stability: another look at MacArthur's original hypothesis. Oikos 33:351-357

Pimm SL (1980) Food web design and the effect of species deletion. Oikos 35:139-149

Pimm SL (1982) Food webs. Chapman and Hall, London. Reprinted from Univ of Chicago Press, Chicago in 2002 with a new Foreword

Pimm SL, Lawton JH (1977) The number of trophic levels in ecological communities. Nature 268:329-331

Pimm SL, Lawton JH (1978) On feeding on more than one trophic level. Nature 275:542-544

Pimm SL, Lawton JH, Cohen JE (1991) Food web patterns and their consequences. Nature 350:669-674

Polis GA (1991) Complex trophic interactions in deserts: an empirical critique of food-web theory. Am Nat 138:123-155

Polis G, McCormick S (1986) Scorpions, spiders and solpugids: predation and competition among distantly related taxa. Oecologia 71:111-116

Polis G, McCormick S (1987) Intraguild predation and competition among desert scorpions. Ecology 68:323-343

Polis GA, Myers CA, Holt RD (1989) The ecology and evolution of intraguild predation: potential competitors that eat each other. Annu Rev Ecol Syst 20:297-330

Post WM, Pimm SI (1983) Community assembly and food web stability. Math Biosci 64:169-192

Poulin R, Morand S (2000) The diversity of parasites. Q Rev Biol 75:277-293

Rejmánek M, Starý P (1979) Connectance in real biotic communities and critical values for stability of model ecosystems. Nature 280:311-313

Roberts A (1974) The stability of a feasible random ecosystem. Nature 251:607-608

Roberts A, Tregonning K (1980) The robustness of natural systems. Nature 288:265-266

Robinson JV, Valentine WD (1979) The concepts of elasticity, invulnerability and invadability. J Theor Biol 81:91-104

Rudolf VHW, Lafferty KD (2011) Stage structure alters how complexity affects stability of ecological networks. Ecol Lett 14:75-79

Sauve AMC, Fontaine C, Thébault E (2014) Structure-stability relationships in networks combining mutualistic and antagonistic interactions. Oikos 123:378-384

Schulze E-D, Mooney HA (eds) (1993) Biodiversity and ecosystem function. Springer-Verlag, Berlin 
Sibly RM, Brown JH, Kodric-Brown A (eds) (2012) Metabolic ecology: a scaling approach. Wiley-Blackwell, Chichester

Solé RV, Montoya JM (2001) Complexity and fragility in ecological networks. Proc R Soc Lond B 268:2039-2045

Sommers HJ, Crisanti A, Sompolinsky H, Stein Y (1988) Spectrum of large random asymmetric matrices. Phys Rev Lett 60:1895-1898

Stewart FM, Levin BR (1973) Partitioning of resources and the outcome of interspecific competition: a model and some general considerations. Am Nat 107:171-198

Stouffer DB, Camacho J, Guimerà R, Ng CA, Amaral LAN (2005) Quantitative patterns in the structure of model and empirical food webs. Ecology 86:1301-1311

Stouffer DB, Camacho J, Amaral LAN (2006) A robust measure of food web intervality. Proc Natl Acad Sci USA 103:19015-19020

Stouffer DB, Rezende EL, Amaral LAN (2011) The role of body mass in diet contiguity and food-web structure. J Anim Ecol 80:632-639

Strogatz SH (2001) Exploring complex networks. Nature 410:268-276

Strong DR, Simberloff D, Abele LG, Thistle AB (eds) (1984) Ecological communities: conceptual issues and the evidence. Princeton Univ Press, Princeton

Sugihara G (1982) Niche hierarchy: structure, organization and assembly in natural communities. Ph.D Dissertation, Princeton Univ, Princeton

Sugihara G (1984) Graph theory, homology and food webs. Proc Symp Appl Math 30:83-101

Suweis S, Grilli J, Martian A (2013) Effects of mixing interaction types on ecological community stability. arXiv:1301.1569 (qbio.PE)

Suweis S, Grilli J, Martian A (2014) Disentangling the effect of hybrid interactions and of the constant effort hypothesis on ecological community stability. Oikos 123:525-532

Tanabe K, Namba T (2005) Omnivory creates chaos in simple food web models. Ecology 86:3411-3414

Tang S, Pawar S, Allesina S (2014) Correlation between interaction strengths drives stability in large ecological networks. Ecol Lett 17:1094-1100

Tao T, Vu V, Krishnapur M (2010) Random matrices: universality of ESDs and the circular law. Ann Probab 38:2023-2065

Thébault E, Fontaine C (2008) Does asymmetric specialization differ between mutualistic and trophic networks? Oikos 117:555-563

Thébault E, Fontaine C (2010) Stability of ecological communities and the architecture of mutualistic and trophic networks. Science 329:853-856

Thébault E, Loreau M (2003) Food-web constraints on biodiversityecosystem functioning relationships. Proc Natl Acad Sci USA 100:14949-14954

Thébault E, Loreau M (2005) Trophic interactions and the relationship between species diversity and ecosystem stability. Am Nat 166:E95-E114

Tilman D (1980) A graphical-mechanistic approach to competition and predation. Am Nat 116:362-393

Tilman D (1996) Biodiversity: population versus ecosystem stability. Ecology 77:350-363

Tilman D (1999) The ecological consequences of changes in biodiversity: a search for general principles. Ecology 80:1455-1474

Tilman D, Downing JA (1994) Biodiversity and stability in grasslands. Nature 367:363-365

Tilman D, Lehman CL, Thomson KT (1997) Plant diversity and ecosystem productivity: theoretical considerations. Proc Natl Acad Sci USA 94:1857-1861

Tilman D, Lehman CL, Bristow CE (1998) Diversity-stability relationships: statistical inevitability or ecological consequence? Am Nat 151:277-282
Tilman D, Isbell F, Cowles JM (2014) Biodiversity and ecosystem functioning. Annu Rev Ecol Evol Syst 45:471-493

Toju H (2015) High-throughput DNA barcoding for ecological network studies. Popul Ecol. doi:10.1007/s10144-014-0472-z

Toju H, Yamamoto S, Sato H, Tanabe AS, Gilbert GS, Kadowaki K (2013a) Community composition of root-associated fungi in a Quercus-dominated temperate forest: "codominance" of mycorrhizal and root-endophytic fungi. Ecol Evol 3:1281-1293

Toju H, Sato H, Yamamoto S, Kadowaki K, Tanabe AS, Yazawa S, Nishimura O, Agata K (2013b) How are plant and fungal communities linked to each other in belowground ecosystem? A massively parallel pyrosequencing analysis of the association specificity of root-associated fungi and their host plants. Ecol Evol 3:3112-3124

Toju H, Yamamoto S, Sato H, Tanabe AS (2013c) Sharing of diverse mycorrhizal and root-endophytic fungi among plant species in an oak-dominated cool-tempereature forest. PLoS ONE 8:e78248

Toju H, Sato H, Tanabe AS (2014a) Diversity and spatial structure of belowground plant-fungal symbiosis in a mixed subtropical forest of ectomycorrhizal and arbuscular mycorrhizal plants. PLoS ONE 9:e86566

Toju H, Guimarães PR, Olesen JM, Thompson JN (2014b) Assembly of complex plant-fungus networks. Nat Commun 5:5273

Tokita K (2004) Species abundance patterns in complex evolutionary dynamics. Phys Rev Lett 93:178102

Tokita K (2006) Statistical mechanics of relative species abundance. Ecol Inform 1:315-324

Tokita K (2015) Analytical theory of species abundance distributions of a random community model. Popul Ecol. doi:10.1007/s10144014-0476-8

Tokita K, Yasutomi A (2003) Emergence of a complex and stable network in a model ecosystem with extinction and mutation. Theor Popul Biol 63:131-146

Tregonning K, Roberts A (1979) Complex systems which evolve towards homeostasis. Nature 281:563-564

van der Heijden MGA, Bardgett RD, van Stralen NM (2008) The unseen majority: soil microbes as drivers of plant diversity and productivity in terrestrial ecosystems. Ecol Lett 11:296-310

Vance RR (1978) Predation and resource partitioning in one predatortwo prey model community. Am Nat 112:797-813

Vitousek PM, Hooper DU (1993) Biological diversity and terrestrial ecosystem biogeochemistry. In: Schulze E-D, Mooney HA (eds) Biodiversity and ecosystem function. Springer-Verlag, Berlin, pp 3-14

Warren PH (1989) Spatial and temporal variation in the structure of a freshwater food web. Oikos 55:299-311

Waser NM, Chittka L, Price MV, Williams NM, Ollerton J (1996) Generalization in pollination systems, and why it matters. Ecology 77:1043-1060

Watts DJ, Strogatz SH (1998) Collective dynamics of 'small-world' networks. Nature 393:440-442

Whitfield J (2006) In the beat of a heart: life, energy, and the unity of nature. Joseph Henry Press, Washington, DC

Wiens JA (1977) On competition and variable environments. Am Sci 65:590-597

Williams RJ, Martinez ND (2000) Simple rules yield complex food webs. Nature 404:180-183

Williams RJ, Martinez ND (2004) Stabilization of chaotic and nonpermanent food-web dynamics. Eur Phys J B 38:297-303

Winemiller KD (1990) Spatial and temporal variation in tropical fish trophic networks. Ecol Monogr 60:331-367

Wootton JT, Emmerson M (2005) Measurement of interaction strength in nature. Annu Rev Ecol Evol Syst 36:419-444

Yodzis P (1981a) The stability of real ecosystems. Nature 289:674-676 
Yodzis P (1981b) The structure of assembled communities. J Theor Biol 92:103-117

Yodzis P, Innes S (1992) Body size and consumer-resource dynamics. Am Nat 139:1151-1175

Yoshino Y, Galla T, Tokita K (2007) Statistical mechanics and stability of a model eco-system. J Stat Mech 2007:P09003
Yoshino Y, Galla T, Tokita K (2008) Rank abundance relations in evolutionary dynamics of random replicators. Phys Rev E 78:031924

Zook AE, Eklof A, Jacob U, Allesina S (2011) Food webs: ordering species according to body size yields high degree of intervality. J Theor Biol 271:106-113 